\title{
Nonexistence of twentieth power residue difference sets
}

\author{
by
}

\author{
Ronald Evans (La Jolla, Cal.)
}

1. Introduction. Let $\mathbb{F}_{p}$ denote the field of $p$ elements, where $p$ is prime. A subset $H \subset \mathbb{F}_{p}$ is a difference set $(\bmod p)$ if there is a fixed integer $\lambda>0$ such that every element of $\mathbb{F}_{p}^{*}$ can be written as a difference of two elements of $H$ in exactly $\lambda$ ways.

Let $H_{k}=H_{k, p}$ denote the set of (nonzero) $k$ th power residues $(\bmod p)$, where $k>1$ and $p$ is a prime of the form $p=k f+1$. If $H_{k}$ is a difference set $(\bmod p)$ ), it is called a $k$ th power residue difference set. If $H_{k} \cup\{0\}$ is a difference set $(\bmod p)$, it is called a modified $k$ th power residue difference set.

By 1953 , the $k$ th power residue and modified $k$ th power residue difference sets had been found for $k=2,4$, and 8 (see [3], [2, Chapter 5]). In the period 1953-1967, the combined work of seven authors showed the nonexistence of such difference sets for all other $k<20$; see the book [1] or [2, Chapter 5] for references.

In 1970, Muskat and Whiteman [4] obtained partial results for the case $k=20$ by showing that $H_{20}$ and $H_{20} \cup\{0\}$ are never difference sets $(\bmod p)$ when 5 is a quartic residue $(\bmod p)$. Regarding the remaining case where 5 is a quartic nonresidue $(\bmod p)$, they wrote: "Efforts to prove that there are no residue difference sets or modified residue difference sets... were unsuccessful." (See [4, p. 215].)

The purpose of this note is to complete the proof that $H_{20}$ and $H_{20} \cup\{0\}$ are never difference sets $(\bmod p)$. This solves Research Problem 11 in $[2, \mathrm{p}$. 497]. (Research Problem 12, the analogous problem for $k=24$, is still open.)

2. Strategy and notation. Let $(i, j), 0 \leq i, j \leq 19$, denote the cyclotomic numbers of order 20 with respect to a fixed primitive $\operatorname{root} g(\bmod p)$, where $p=20 f+1$. Assume for the purpose of contradiction that $H_{20}$ or $H_{20} \cup\{0\}$ is a difference set $(\bmod p)$. Then ([3], [4, p. 214]) $f$ is odd, 5 is a

1991 Mathematics Subject Classification: Primary 05B10. 
quartic nonresidue $(\bmod p)$, and

$$
\begin{aligned}
& 1600(0,0)=4 p-80-4 \nu^{2}, \\
& 1600(i, 0)=4 p-76+8 \nu, \quad 1 \leq i \leq 9,
\end{aligned}
$$

where

$$
\nu= \begin{cases}-1 & \text { if } H_{20} \text { is a difference set, } \\ 19 & \text { if } H_{20} \cup\{0\} \text { is a difference set. }\end{cases}
$$

The cyclotomic numbers in (1) and (2) are expressed in the tables of [4] as linear combinations of $p, 1, c, d, x, u, v, w$, and $d_{j}(0 \leq j \leq 19)$, where these integral parameters are as defined in [4]. In particular (see [4, eqs. (4.14), (4.1), (2.18), (2.17)]),

$$
\begin{gathered}
p=c^{2}+5 d^{2} \\
16 p=x^{2}+125 w^{2}+50 u^{2}+50 v^{2} \\
x \equiv 1(\bmod 5) \\
x w=v^{2}-u^{2}-4 u v \\
p=\left|\sum_{j=0}^{9} d_{j} \zeta^{j}\right|^{2}, \quad \text { where } \zeta=\exp (2 \pi i / 20),
\end{gathered}
$$

and

$$
d_{j}=-d_{j-10} \quad \text { for } 10 \leq j \leq 19 .
$$

If we formally expand

$$
\sum_{j=0}^{9} d_{j} \zeta^{j} \sum_{j=0}^{9} d_{9-j} \zeta^{j}-p \zeta^{9},
$$

and then make the substitutions

$$
\begin{aligned}
\zeta^{k}=-\zeta^{k-10} & (10 \leq k \leq 18), \\
\zeta^{9}=\zeta^{7}-\zeta^{5}+\zeta^{3}-\zeta, & \zeta^{8}=\zeta^{6}-\zeta^{4}+\zeta^{2}-1,
\end{aligned}
$$

we obtain the sum

$$
\sum_{r=0}^{7} G_{r} \zeta^{r}
$$

where

$$
\begin{aligned}
& G_{0}=-2 \sum_{j=0}^{9} d_{j} d_{j+1}, \\
& G_{1}=p-\sum_{j=0}^{9} d_{j}^{2}-\sum_{j=0}^{9} d_{j} d_{j+2},
\end{aligned}
$$




$$
\begin{aligned}
G_{2} & =\sum_{j=0}^{9} d_{j} d_{j+1}-\sum_{j=0}^{9} d_{j} d_{j+3}, \\
G_{3} & =\sum_{j=0}^{9} d_{j}^{2}-p-\sum_{j=0}^{9} d_{j} d_{j+4},
\end{aligned}
$$

$G_{4}=G_{0} / 2, G_{5}=-G_{3}, G_{6}=-G_{2}-G_{0}$, and $G_{7}=-G_{1}$. By (8), the sums in (10) and (11) vanish, and thus

$$
G_{0}=G_{1}=G_{2}=G_{3}=0
$$

since $\left\{1, \zeta, \zeta^{2}, \ldots, \zeta^{7}\right\}$ is a basis for $\mathbb{Q}(\zeta)$ over $\mathbb{Q}$.

Our strategy is to obtain the desired contradiction by showing that (2) is inconsistent with (4)-(7) and (16). As was pointed out in [4, p. 215], we need to consider just two cases. The first case is

$$
\operatorname{ind}_{g} 2 \equiv 5(\bmod 10), \quad c \equiv 6(\bmod 10)
$$

and the second case is

$$
\text { ind }_{g} 2 \equiv 1(\bmod 10), \quad c \equiv 6(\bmod 10) .
$$

These cases are discussed in Sections 3 and 4, respectively.

3. The case $\operatorname{ind}_{g} 2 \equiv 5(\bmod 10), c \equiv 6(\bmod 10)$. View the last nine rows of Table 4 in [4, pp. 212-213] as a system of nine linear equations in the nine variables $d_{0}, d_{4}, d_{8}, d_{12}, d_{16}, d_{1}, d_{5}, d_{9}$, and $d_{13}$. Replace each $1600(i, 0)$ in this system by $4 p-76+8 \nu$ (see (2)). Using Maple to solve this system, we obtain expressions for the nine variables as linear combinations of $\nu, c, d, x, w, u, v, d_{17}$ over $\mathbb{Q}$. For example, $d_{0}=-3(x+\nu) / 5$. Then from (12)-(15), each of $G_{0}, G_{1}, G_{2}$, and $G_{3}$ can be written as a quadratic polynomial in $p, \nu, c, d, x, w, u, v$ over $\mathbb{Q}\left(d_{17}\right.$ does not appear). These polynomials are rather cumbersome (e.g., $G_{1}$ and $G_{3}$ each have 18 terms) and so we do not write them explicitly here. A Maple program which produces these polynomials is currently available upon request.

Reducing $(2)$ and $(5)(\bmod 25)$, and using $(6)$, we deduce that

$$
x \equiv 5-\nu(\bmod 25) \text {. }
$$

Also, by (3) and (17),

$$
5 \nu \equiv-5(\bmod 25), \quad 5 c \equiv 5(\bmod 25) .
$$

We cannot have $u=v=0$, in view of (5) and (7). Hence one can define

$$
u_{0}=u / \operatorname{gcd}(u, v), \quad v_{0}=v / \operatorname{gcd}(u, v) .
$$

Dividing the equality $0=G_{0}-G_{2}$ by $\operatorname{gcd}(u, v)$ and then reducing $\bmod 25$, we obtain

$$
0 \equiv 18 x u_{0}+x v_{0}+5 c u_{0}+10 c v_{0}+3 \nu u_{0}+21 \nu v_{0}(\bmod 25) .
$$


Substituting in the value of $x$ given by (19), and then making the substitutions for $5 \nu$ and $5 c$ given by (20), we obtain

$$
0 \equiv 10 u_{0}-5 v_{0}(\bmod 25) .
$$

Reduction of the equality $0=G_{1}+G_{3}$ modulo 25 yields, after the substitution of $x$ from (19),

$$
0 \equiv 20 \nu w+10 c w+5 d+20 u v+20 v^{2}+5 u^{2}(\bmod 25) .
$$

After substitutions from (20) and (22), this becomes

$$
0 \equiv 5 d-10 w(\bmod 25) \text {. }
$$

From (7) and (22), we see that $5 \mid x w$, so that by (6), $5 \mid w$. Thus by (23),

$$
d \equiv w \equiv 0(\bmod 5) .
$$

Dividing the equality $0=G_{0}+G_{2}$ by $\operatorname{gcd}(u, v)$ and then reducing $\bmod$ 25 , we obtain, after the substitution of $x$ from (19),

$$
0 \equiv 20 u_{0}+10 \nu u_{0}+5 c u_{0}+20 d u_{0}+15 d v_{0}+15 v_{0}(\bmod 25) .
$$

After substitutions from (20), (22), and (24), this becomes

$$
0 \equiv 20 u_{0}(\bmod 25) \text {. }
$$

Then $5 \mid u_{0}$, which contradicts $(22)$, because $\operatorname{gcd}\left(u_{0}, v_{0}\right)=1$ by (21). This completes the proof that $H_{20}$ and $H_{20} \cup\{0\}$ are never difference sets in the case (17).

4. The case $\operatorname{ind}_{g} 2 \equiv 1(\bmod 10), c \equiv 6(\bmod 10)$. We express $G_{0}, G_{1}$, $G_{2}$ and $G_{3}$ as quadratic polynomials just as in Section 3, except that instead of using the last nine rows of Table 4, we use rows $21,22,38,40,52,55,63$, 67, 71 of Table 1 in [4, pp. 204-207]. The polynomials are more complicated than those in Section 3; for example, $G_{3}$ has 28 terms instead of 18 .

Since $f$ is odd, $p \equiv 5(\bmod 8)$. Since $c$ is even by $(18)$, and $p=c^{2}+5 d^{2}$ by (4), it follows that $4 \mid c$. Write

$$
c=4 c_{2},
$$

where $c_{2}$, as well as each parameter introduced below, is integral. Since $\nu$ is -1 or 19 by (3), write

$$
\nu=-1+4 \nu_{2} .
$$

By (2),

$$
p=19-2 \nu+16 p_{4} .
$$

From [2, Theorem 3.7 .9 , p. 135], we can write

$$
\begin{aligned}
& x=1+2 x_{1}, \\
& u=2 u_{1},
\end{aligned}
$$




$$
v=x+u+4 s_{2} .
$$

Further, from [2, eq. (3.7.46), p. 135],

$$
w=x-2 u+8 t_{3} \text {. }
$$

Write

$$
E:=-x w+v^{2}-u^{2}-4 u v,
$$

so that $E=0$ by $(7)$.

From $E / 8 \equiv 0(\bmod 2)$, we see that $t_{3}+s_{2}$ is even. From $4 G_{1} \equiv 0(\bmod 2)$, we see that $1+u_{1}+s_{2}$ is even. Thus

$$
\begin{aligned}
& s_{2}=1+u_{1}+2 s_{3}, \\
& t_{3}=1+u_{1}+2 t_{4} .
\end{aligned}
$$

We now consider separately the two cases $d \equiv \pm 1(\bmod 4)$.

CAse $1: d \equiv-1(\bmod 4)$. In this case, write

$$
d=-1+4 d_{2} .
$$

From $E / 16 \equiv 0(\bmod 2)$, we see that $1+s_{3}+t_{4}$ is even. From $G_{0} / 2 \equiv$ $0(\bmod 2)$, we see that $x_{1} s_{3}+u_{1}+t_{4}$ is even. From $G_{1} \equiv 0(\bmod 2), x_{1} s_{3}+u_{1}$ is even, so that $t_{4}$ is even and $s_{3}$ is odd. From $G_{2} \equiv 0(\bmod 2), x_{1} u_{1}+x_{1}$ is even. From $G_{3} \equiv 0(\bmod 2), x_{1} u_{1}+x_{1}+u_{1}$ is even. Combining these five results, we can write

$$
\begin{aligned}
t_{4} & =2 t_{5}, \\
s_{3} & =1+2 s_{4}, \\
u_{1} & =2 u_{2}, \\
x_{1} & =2 x_{2} .
\end{aligned}
$$

From these formulas we arrive at

$$
G_{1} / 2+G_{2} / 2+E / 32 \equiv 1(\bmod 2),
$$

which is a contradiction, since $G_{1}=G_{2}=E=0$.

CASE $2: d \equiv 1(\bmod 4)$. In this case, $d \equiv-\nu(\bmod 8)$, since by $(7),(25)$, and $(27), 19-2 \nu \equiv p \equiv 5 d^{2}(\bmod 16)$. Thus write

$$
d=-\nu+8 d_{3} .
$$

From $E / 16 \equiv 0(\bmod 2)$, we see that $1+s_{3}+t_{4}$ is even. From $G_{0} / 2 \equiv$ $0(\bmod 2), x_{1} s_{3}$ is even. From $G_{1} \equiv 0(\bmod 2), u_{1}$ is even. From $G_{2} \equiv$ $0(\bmod 2), 1+x_{1} t_{4}$ is even. From $G_{3} \equiv 0(\bmod 2), x_{1}+x_{1} t_{4}$ is even. Combining these five results, we can write

$$
\begin{aligned}
& t_{4}=1+2 t_{5}, \\
& s_{3}=2 s_{4},
\end{aligned}
$$




$$
\begin{aligned}
& u_{1}=2 u_{2}, \\
& x_{1}=2 x_{2}+1 .
\end{aligned}
$$

From $E / 32 \equiv 0(\bmod 2), 1+u_{2}+t_{5}+s_{4}$ is even. From $G_{0} / 4 \equiv 0(\bmod 2)$, $s_{4}+\nu_{2}$ is even. From $G_{1} / 2 \equiv 0(\bmod 2), \nu_{2}+u_{2}+x_{2}+t_{5}+s_{4}$ is even. Combining these three results, we can write

$$
\begin{aligned}
& s_{4}=-\nu_{2}+2 s_{5}, \\
& x_{2}=1-\nu_{2}+2 x_{3}, \\
& t_{5}=1-\nu_{2}+u_{2}+2 t_{6} .
\end{aligned}
$$

From these formulas, we arrive at

$$
G_{0} / 8+G_{1} / 4+G_{3} / 4 \equiv 1(\bmod 2)
$$

which is a contradiction, since $G_{0}=G_{1}=G_{3}=0$.

The contradictions obtained in Cases 1 and 2 complete the proof that $H_{20}$ and $H_{20} \cup\{0\}$ are never difference sets in the case (18).

\section{References}

[1] L. D. Baumert, Cyclic Difference Sets, Lecture Notes in Math. 182, Springer, Berlin, 1971.

[2] B. C. Berndt, R. J. Evans and K. S. Williams, Gauss and Jacobi Sums, Wiley, New York, 1998.

[3] E. Lehmer, On residue difference sets, Canad. J. Math. 5 (1953), 425-432.

[4] J. B. Muskat and A. L. Whiteman, The cyclotomic numbers of order twenty, Acta Arith. 17 (1970), 185-216.

Department of Mathematics University of California, San Diego

La Jolla, California 92093-0112

U.S.A.

E-mail: revans@ucsd.edu 